\title{
An approach for testing asynchronous communicating systems
}

\author{
Myungchul Kim", Samuel T. Chanson"*, Sungwon Kang*, \\ Jaehwi Shin ${ }^{*}$ \\ * Korea Telecom $R \&$ D Group \\ Sochogu, Seoul, Korea 137-140 \\ \{mckim,kangsw,jhshin\}@sava.kotel.co.kr \\ ${ }^{*}$ *Hong Kong Univ. of Science \& Technology \\ Clear Water Bay, Kowloon, Hong Kong \\ chanson@cs.ust.hk
}

\begin{abstract}
This paper studies the problem of testing concurrent systems as blackboxes described in asynchronous Communicating Finite State Machines. We present an approach to derive test cases in a succinct and formal way for the concurrent systems. The approach avoids the state space explosion problem by introducing a causality relation model to express true concurrency. A new test architecture for executing the test suite is also described.
\end{abstract}

\section{Keywords}

Protocol Testing, Concurrent System, Conformance Testing, Logical Time.

\section{INTRODUCTION}

Concurrent behavior of a distributed system is difficult to specify and analyze. In order to specify the concurrent behavior of communication protocols and services in a distributed system, formal description techniques such as Communicating Finite State Machines (CFSMs), Estelle, SDL, LOTOS, and others have been developed. These techniques allow interactions among multiple machines (or modules or processes) to be specified. In order to increase confidence in protocol products made according to international standards, various protocol (conformance and interoperability) testing methods have been developed and 
applied. In the ISO conformance testing methodology, an Implementation Under Test (IUT) is viewed as a blackbox for testing purposes (ISO, 1994). Up until now, most work on automatic test case generation has been focused on single machine specifications even though the implementation may consist of multiple machines. Typically, a preprocessing stage is used to translate the multiple machines into a single system in order to obtain a specification with sequential behavior because we have a variety of testing methods for sequential systems and understand sequential behavior better. In this stage, the "state space explosion" problem often occurs.

In this paper, we present a succinct and formal methodology using true concurrency for generating test cases from a concurrent system described as asynchronous CFSMs and then applying the test cases to the concurrent system. Briefly speaking, the approach is to adopt techniques for distributed systems and apply them into protocol conformance testing on a concurrent system. The difficulty in testing a concurrent system is occurred by intentional nondeterminism existing in the specification and unintentional nondeterminism either arising from the concurrent environment or due to the blackbox approach (Kim, 1995). Our work is to deal with the unintentional nondeterminism in asynchronous CFSMs.

Related papers exist in the software engineering field such as (Young, 1988, Taylor, 1992, Tai, 1995). In the protocol engineering area, there are a few papers such as (Araka ,1991, Lee, 1993, Caval, 1994, Luo 1994, Ulric 1995). Our work is different from the existing work from the fact that our work aims at testing asynchronous CFSMs as treating them blackboxes and is a non-heuristic approach using a true concurrency model. It is an effort for coping with inherently difficult issues in testing a concurrent system.

The rest of the paper is organized as follows; Section 2 surveys some related work and Section 3 summarizes logical clocks (Lampo, 1978, Rayna, 1992a) for maximizing concurrency which is a crucial step in testing concurrent systems. Section 4 presents the model we propose. In addition, test case generation as well as application of the model is described. The section also gives definitions such as Observability Rate and Controllability Rate. Finally Section 5 concludes the paper.

\section{RELATED WORK}

Our work aims at testing asynchronous CFSMs which are treated as blackboxes. We provide a non-heuristic approach which uses a true concurrency model. By comparison, most of the existing work in software engineering such as (Young, 1988, Taylor, 1992, Tai, 1995 ) is based on synchronous communicating programs where the internal codes are accessible (whitebox approach).

The area of protocol testing has more similarity to our work, thus the following is a brief survey of some related papers on testing concurrent systems in protocol testing. (Araka, 1991) proposes a test case generation method for concurrent programs as a blackbox. They generate the control flow graph representing the global behavior of a given concurrent program and then apply conventional test case generation methods on the control flow graph. To avoid state space explosion, blackbox equivalence between system behavior is introduced. The approach is a modified technique from (Kajiw, 1985), and works for asynchronous CFSMs as blackboxes. However, the method is not formally presented.

Lee et. al (Lee, 1993) present an approach for conformance testing of protocols specified as a collection of synchronous CFSMs. The approach has two parts: pruning and a guided random walk procedure. This allows some parts of the behavior space to be removed from consideration. Also, the model is based on interleaving in order to explore the state space. 
(Caval, 1994) presents a test sequence generation method based on Unique Input/Output sequences and signatures in a framework which allows conformance test sequences to be obtained from LOTOS specifications. The model is for synchronous communicating systems.

Luo et al. (Luo, 1994) present a model of generating test sequences for concurrent systems and communication protocol model as communicating nondeterministic finite state machines (CNFSMs). After CNFSMs are transformed into a single nondeterministic finite state machine (NFSM) by reachability analysis, test sequences are generated from the resulting NFSM using the generalized Wp-method. The approach meets the state space explosion problem easily because it adopts the reachability analysis in the transformation phase.

In (Ulric, 1995), an approach to deriving a concurrent transition tour as a test suite from multi-module specifications described by a restricted class of Calculus of Communicating Systems is presented. This approach avoids the state space explosion problem by introducing a true concurrency model. Thus this model is for synchronous communicating systems and uses some heuristics in terms of the order of composition.

The work in (Verha, 1992) shows that test cases generated using algorithms for synchronous communication can not be used for asynchronous systems. Thus, our work is different from all of work listed above in a sense that our work aims at testing asynchronous CFSMs as treating them blackboxes and is a non-heuristic approach using a true concurrency model.

\section{BACKGROUND: LOGICAL CLOCKS AND MAXIMIZING CONCURRENCY}

Since Lamport's pioneering work (Lampo, 1978), there has been considerable research on concurrency, logical clocks and global states for distributed systems (Fidge, 1991, Matte, 1988, Charr, 1989, Rayna, 1992a, Rayna, 1992b, Kim, 1993). The logical clock is used to determine whether or not two events are concurrent. The concurrency relation is then used to compute global states.

Given a complete specification, causality relationship of events may be obtained readily using a concurrency model (Kim, 1993). Here, we adopt logical clocks (Lampo, 1978, Rayna, 1992a) to incorporate the duration of an operation in the model even though the causality relation is known and does not have to be computed on-the-fly. The purpose is to handle unintentional nondeterminism either arising in the concurrent environment or due to the blackbox approach in asynchronous CFSMs.

\subsection{Logical clocks}

Lamport showed that distributed asynchronous executions may be characterized by a partial order relation on the events produced. The relation, called the causality relation (or happened before relation) and denoted by $\rightarrow$, is defined in the following way (Lampo, 1978). Let $\mathrm{E}$ be a set of events produced by the execution of a distributed system. For $\mathrm{e}, \mathrm{e}^{\prime}$, $\mathrm{e}^{\prime \prime} \in \mathrm{E}, \mathrm{e} \rightarrow \mathrm{e}^{\prime}$ holds if

1. e and $\mathrm{e}^{\prime}$ are events in the same process and e precedes $\mathrm{e}^{\prime}$,

2. $\mathrm{e}$ is a send event and $\mathrm{e}^{\prime}$ is the corresponding receive event, or

3. there exists $\mathrm{e}^{\prime \prime}$ such that $\mathrm{e} \rightarrow \mathrm{e}^{\prime \prime}$ and $\mathrm{e}^{\prime \prime} \rightarrow \mathrm{e}^{\prime}$. 
Two events $\mathrm{e}$ and $\mathrm{e}^{\prime}$ are said to be causally related if $\mathrm{e} \rightarrow \mathrm{e}^{\prime}$ or $\mathrm{e}^{\prime} \rightarrow \mathrm{e}$ holds. If neither $\mathrm{e}$ $\rightarrow \mathrm{e}^{\prime}$ nor $\mathrm{e}^{\prime} \rightarrow \mathrm{e}$ holds, these events are concurrent (or mutually independent), denoted by $\mathrm{e}$ $\| \mathrm{e}^{\prime}$.

According to the above principles, a number of timestamping mechanisms are devised such as linear time, vector time, and matrix time (Rayna, 92a). Linear time $c_{i}$ associated with process $P_{i}$, for example, can be computed as follows:

- When $P_{i}$ executes an internal event or a send event, the time is computed by $c_{i}=c_{i}+d$. In case of a send event, the message carries the updated $c_{i}$ value as the timestamp.

- When $P_{i}$ executes a receive event where the message contains timestamp $t s$, the time is computed by $\mathrm{c}_{\mathrm{i}}=\max \left(\mathrm{c}_{\mathrm{i}}, \mathrm{ts}\right)+\mathrm{d}$.

The value $d$ represents the duration of the corresponding operation.

In case of vector time (Fidge, 91, Matte, 1988), the logical global time represented by an $n$-dimensional vector $v_{i}[1 . . n]$ is associated with each process $P_{i}$ where $n$ is the number of counters which is equal to the number of processes. The vector $v_{t}[j]$ denotes the $j$-th counter maintained by process $P_{i}$. All values in $v_{i}[1 . . n]$ are initialized to zero. The principle of the technique is the following:

- When $P_{i}$ executes an internal event, $v_{i}$ is advanced by setting $v_{i}[i]:=v_{i}[i]+d$.

- When $P_{i}$ executes a send event, $v t_{i}$ is advanced by setting $v t_{i}[i]:=v t_{i}[i]+d$. The message carries the updated $v t_{\mathrm{i}}$ value.

- When $P_{\mathrm{i}}$ executes a receive event and reads a message containing $\mathrm{vt}_{\mathrm{j}}, \mathrm{vt}_{\mathrm{i}}$ is advanced by setting $\mathrm{vt}_{\mathrm{i}}[\mathrm{k}]:=\max \left(\mathrm{vt}_{\mathrm{i}}[\mathrm{k}], \mathrm{vt}_{\mathrm{j}}[\mathrm{k}]\right), 1 \leq \mathrm{k} \leq \mathrm{n}$, and then $\mathrm{vt}_{\mathrm{i}}[\mathrm{i}]:=\mathrm{vt}_{\mathrm{j}}[\mathrm{i}]+\mathrm{d}$.

If event $x$ at site $i$ and event $y$ at site $j$ are timestamped respectively by $v h$ and $v k$, we have:

$$
\begin{aligned}
& x \rightarrow y \text { if and only if }(v h[i] \leq v k[i] \text { and } v h[j]<v k[j]) \ldots(1) \\
& x \| y \text { if and only if vh[i] }>v k[i] \text { and } v h[j]<v k[j]
\end{aligned}
$$

A computation may be graphically displayed in a time-event sequence diagram, such as the one shown in Figure 1. The origin of an arrow represents a send event and the head of an arrow indicates a receive event. Figure 1 shows an example of vector clocks progress with the value $\mathrm{d}=1$. The values of $v t$ are represented by $\left(i_{1}, i_{2}, i_{3}\right)$.

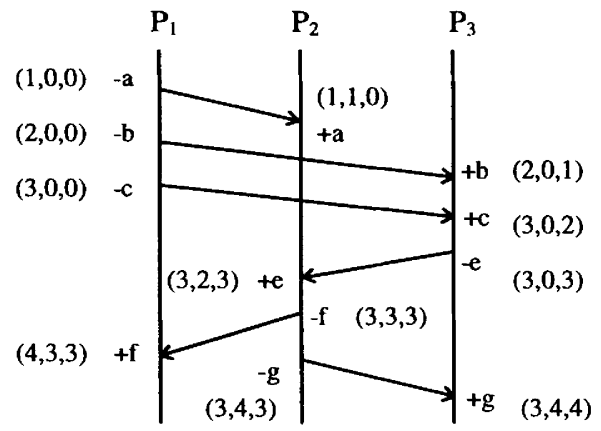

Figure 1 A computation. 
Using (1) and (2) given above, we can decide whether two events are concurrent or not. For example, event ' $-b$ ' precedes event ' $+b$ ' and ' $+c$ ' because of (1) and event ' $-b$ ' and ' $+a$ ' are concurrent because of (2). Also note that not only are ' $-b$ ' and ' $+a$ ' concurrent because of (2) but ' $+a$ ' and '-c' are also concurrent by (2) event though '- $b \rightarrow-c$ '.

\subsection{Maximizing concurrency}

Any analysis technique should be independent of real time effects, such as system load and processor speed. To abstract away from any particular machine executing a distributed program, we assume that each event consumes approximately the multiple amount of a time unit. By the assumption, we can set durations of events to any integer numbers. These assumptions can be achieved by adjusting the granularity of the events. Such an approach allows us to precisely characterize the causality constraints inherent within a distributed computation without being bothered by the perturbations caused by a given implementation.

In the model given in (Rayna, 1992b), the execution time based on time units can be computed using linear logical time described in the previous section. For simplicity, we further assume that the duration of each event is a unit time. Based on this, we draw a timeevent sequence diagram by aligning all events with the same logical time represented by the same dotted row, as shown in Figure 2. Those events are concurrent ones which can be represented by ' $\|$ ' relation defined in previous section. Then, the time-event sequence diagram for a given computation is unique and minimal in terms of the number of time units (Rayna, 1992b). In the figure, synchronization delays represented by circles occur where events are not produced by processes.

For example, the linear logical time of event ' $\mathrm{e}$ ' is ' 5 '. In Figure 2, the logical time of every event is increased by ' 1 ' and every event is aligned with respect to the logical time for later usage.

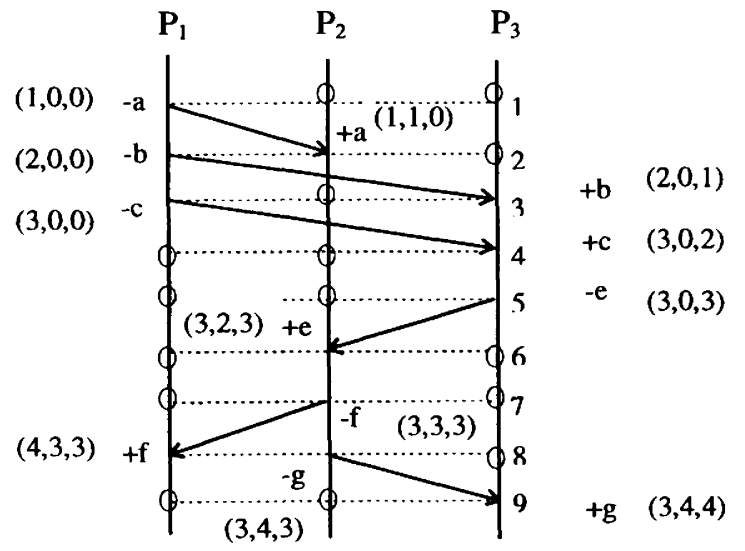

Figure 2 A computation with linear logical clock. 


\section{FORMAL MODEL}

In this section, we propose a model to derive test cases from a concurrent system in a succinct and formal way. The concurrent system is represented in asynchronous CFSMs and treated as blackboxes.

\subsection{System modeling}

A concurrent system consists of a number of sequential systems. Each sequential system is modeled as a Finite State Machine (FSM). The environment (e.g., operators, testers, and/or subsystems which are not under test) of a concurrent system is also modeled in term of FSMs. It is assumed that there is no global physical clock; that is, the system model is asynchronous. FSMs exchange messages to communicate and synchronize with each other. The underlying message passing system provides asynchronous communication and is assumed to be reliable; that is, there is no message loss, distortion or duplication.

An event is the atomic execution of an operation within a system. There are three types of events:

- Internal event which models the execution of an internal operation of an FSM.

- Send event which models a message send operation of an FSM.

- Receive event which models a message receive operation of an FSM.

We assume that a concurrent system consists of subsystems with no access to global variables. Thus, there are no dataraces in this model. Also each subsystem is assumed to be a blackbox. Therefore, only communication events such as message send and receive events are considered. Also a send (or receive) event takes a logical unit time as we assumed in Section 3.

Definition 1: A Global Event (GE) is defined as a set of events occurred at the same time in all FSMs of a concurrent system. Namely, $G E=\left(e_{1}, \ldots, e_{i}, \ldots, e_{n}\right)$ where $1 \leq i \leq n, n$ is equal to the number of FSMs, and $e_{i}$ is an event of $i$-th FSM.

An element, $e_{i}$, of GE is null if $i$-th FSM is idle. Note that GE does not include the state of queues because events are external behavior abstracted away from internal behavior such as queue operations.

The GE consists of send and/or receive events of one or more subsystem because we allow true concurrency. A true concurrency can be expressed using a causality relation model. The causality relation model provides an answer to the question whether events occur independently (Visse, 1990). In order to verify the behavior of a concurrent system, techniques commonly employed are based upon interleaving of actions among subsystems in the concurrent system, thereby leading to state space explosion when the number of subsystems is large. In fact, we really do not need interleaving for those concurrent actions which do not affect each other (e.g., via datarace). It is possible to use a causality relation model to express true concurrency instead of using interleaving in order to test concurrent systems. We propose an approach to test concurrent behavior as it is.

\subsection{Generation of Concurrent Paths}

For simplicity, we shall consider the second visit to the initial state of an FSM as termination. A path is a sequence of events performed by the FSM starting and ending at 
the same initial state in a normal case. From the paths obtained from enumeration every possible path of an FSM, we select a set of paths among the FSMs communicating with one another. For the set of paths, we can make a vector of matching event sequences (in a path) ordered with respect to each FSM.

Definition 2: Path[i] is a set of event sequences, tours, that can be performed by i-th FSM, i.e. $\operatorname{Path}[\mathrm{i}]=\{\operatorname{Tour}(i, 1), \ldots, \operatorname{Tour}(i, y), \ldots\}$ where $\operatorname{Tour}(i, y)$ is $y$-th tour and is denoted by $\mathrm{e}^{i}$ $\rightarrow \mathrm{e}^{2} \rightarrow \ldots \rightarrow \mathrm{e}^{\mathrm{m}} \ldots \rightarrow \mathrm{e}^{\mathrm{M}}$ for some natural number $\mathrm{M}$.

Definition 3: A Concurrent Path (CP) is a tour (or event sequence) vector among FSMs. Namely, $\mathrm{CP}_{\mathrm{k}}=\langle\operatorname{Tour}(1, \mathrm{x}), \ldots, \operatorname{Tour}(\mathrm{i}, \mathrm{y}), \ldots, \operatorname{Tour}(\mathrm{n}, \mathrm{z})\rangle$ where $1 \leq \mathrm{i} \leq \mathrm{n}, \mathrm{n}$ is equal to the number of FSMs, and $\mathrm{k}, \mathrm{x}, \mathrm{y}$ and $\mathrm{z}$ are some natural numbers.

CPs are obtained from Path $1 \times \ldots \times$ Path $_{i} \times \ldots \times$ Path $_{n}$ where the operator ' $\times$ ' is the Cartesian product such that a send event is matched to its receive event in chronological order in every tour of Paths in FSMs.

An element, Tour(i,y), of CP is null if $i$-th FSM is idle. If there is no CP, $k$ in Definition 3 becomes ' 0 '. The meaning of CP is the same as Multilogue in (Araka, 1991, Kajiw, 1985).

The CP begins with the initial state and ends with a node which is not the initial node if there is a deadend such as deadlock, unspecified reception, and so forth. In this case, CP could contain some unpaired messages. If there is no deadend, the $\mathrm{CP}$ eventually returns to the initial state.
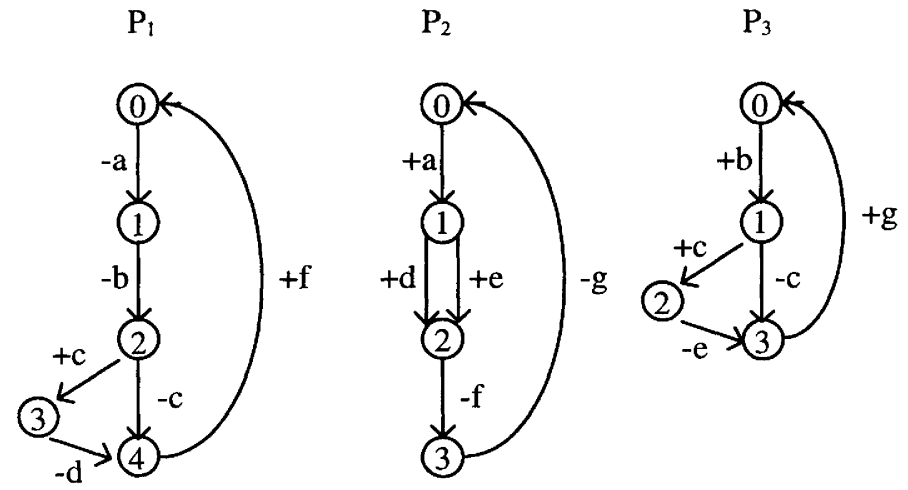

Figure 3 Example of a concurrent system.

For the example shown in Figure 3 which was adopted from (Kajiw, 1985), we can generate a set of individual Paths as follows:

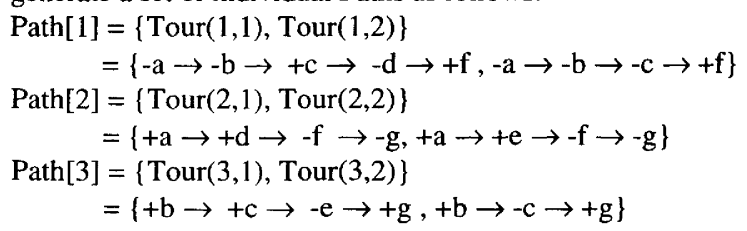


The cross product of three paths and two tours per path gives eight concurrent paths. Let $<\mathrm{x}, \mathrm{y}, \mathrm{z}>$ represent a CP consisting of $<\operatorname{Tour}(1, \mathrm{x})$, $\operatorname{Tour}(2, \mathrm{y})$, $\operatorname{Tour}(3, \mathrm{z})>$ and 'Combi $i$ ' mean the $\mathrm{i}$-th combination of tours among paths. From this, we can obtain eight combinations:

Combi_1 $1=<1,1,1>=\mathrm{CP}_{3}$

Combi_2 $=<1,1,2\rangle=\mathrm{CP}_{1}$

Combi_3 $=<1,2,1\rangle=\mathrm{CP}_{3}$

Combi_ $4=\langle 1,2,2\rangle=\mathrm{CP}_{5}$

Combi_5 $=<2,1,1>=\mathrm{CP}_{6}$

Combi_6 $=<2,1,2\rangle=\mathrm{CP}_{4}$

Combi_ $7=<2,2,1>=\mathrm{CP}_{2}$

Combi_ $8=\langle 2,2,2\rangle=\mathrm{CP}_{4}$

$\left.\mathrm{CP}_{1}=<-\mathrm{a} \rightarrow-\mathrm{b} \rightarrow+\mathrm{c} \rightarrow-\mathrm{d} \rightarrow+\mathrm{f},+\mathrm{a} \rightarrow+\mathrm{d} \rightarrow-\mathrm{f} \rightarrow-\mathrm{g},+\mathrm{b} \rightarrow-\mathrm{c} \rightarrow+\mathrm{g}\right\rangle$

$\mathrm{CP}_{2}=<-\mathrm{a} \rightarrow-\mathrm{b} \rightarrow-\mathrm{c} \rightarrow+\mathrm{f},+\mathrm{a} \rightarrow+\mathrm{e} \rightarrow-\mathrm{f} \rightarrow-\mathrm{g},+\mathrm{b} \rightarrow+\mathrm{c} \rightarrow-\mathrm{e} \rightarrow+\mathrm{g}>$

$\mathrm{CP}_{3}=<-\mathrm{a} \rightarrow-\mathrm{b} \rightarrow+\mathrm{c},+\mathrm{a} \rightarrow+\mathrm{e},+\mathrm{b} \rightarrow+\mathrm{c}>$

$\mathrm{CP}_{4}=<-\mathrm{a} \rightarrow-\mathrm{b} \rightarrow-\mathrm{c} \rightarrow+\mathrm{f},+\mathrm{a} \rightarrow+\mathrm{d},+\mathrm{b} \rightarrow-\mathrm{c} \rightarrow+\mathrm{g}>$

$\left.\mathrm{CP}_{5}=<-\mathrm{a} \rightarrow-\mathrm{b} \rightarrow+\mathrm{c} \rightarrow-\mathrm{d} \rightarrow+\mathrm{f},+\mathrm{a} \rightarrow+\mathrm{e},+\mathrm{b} \rightarrow-\mathrm{c} \rightarrow+\mathrm{g}\right\rangle$

$\mathrm{CP}_{6}=<-\mathrm{a} \rightarrow-\mathrm{b} \rightarrow-\mathrm{c} \rightarrow+\mathrm{f},+\mathrm{a} \rightarrow+\mathrm{d},+\mathrm{b} \rightarrow+\mathrm{c} \rightarrow-\mathrm{e} \rightarrow+\mathrm{g}>$

Note that $\mathrm{CP}_{3}, \mathrm{CP}_{4}, \mathrm{CP}_{5}$, and $\mathrm{CP}_{6}$ reach deadends because these $\mathrm{CPs}$ do not end with the initial node.

Let us look at the traditional reachability analysis. It can be considered that the analysis consists of two levels of enumeration on:

1. paths among FSMs,

2. and events among the paths obtained.

Level 1 of enumeration in the traditional reachability analysis corresponds to the computation of CPs defined in Definition 3. In this level, we may encounter "state space explosion" which is not handled in this paper. The next step is trying to solve the "state space explosion" occurring in events enumeration (in level 2) among the paths obtained. Traditional reachability analysis enumerates one path out of a set of system (or FSM) interactions based on interleaving of events and each of which is tested individually. In this way, the global state space grows rapidly as the number and the complexity of the subsystems increase.

Furthermore, loops in a path could also lead to state space explosion since the potential number of distinct paths is infinite. Practically we can limit the number of times a loop is traversed.

\subsection{Generation of Minimal Causality Path}

Let's enumerate every possible event sequence by interleaving satisfying the following Concurrent Path:

$\mathrm{CP}_{2}=<-\mathrm{a} \rightarrow-\mathrm{b} \rightarrow-\mathrm{c} \rightarrow+\mathrm{f},+\mathrm{a} \rightarrow+\mathrm{e} \rightarrow-\mathrm{f} \rightarrow-\mathrm{g},+\mathrm{b} \rightarrow+\mathrm{c} \rightarrow-\mathrm{e} \rightarrow+\mathrm{g}>$.

In the $\mathrm{CP}$, there are constraints imposed by causal relationships among the events. These consists of two kinds: ordering imposed by event sequences in $\mathrm{CP}$ and ordering imposed by matching send and receive events. So far in $\mathrm{CP}_{2}$, we have the following constraints of the first kind: 
- $-\mathrm{a} \rightarrow-\mathrm{b} \rightarrow-\mathrm{c} \rightarrow+\mathrm{f}$,

- $+\mathrm{a} \rightarrow+\mathrm{e} \rightarrow-\mathrm{f} \rightarrow-\mathrm{g}$, and

- $+\mathrm{b} \rightarrow+\mathrm{c} \rightarrow-\mathrm{e} \rightarrow+\mathrm{g}$.

The constraints of the second kind are: $-\mathrm{a} \rightarrow+\mathrm{a},-\mathrm{b} \rightarrow+\mathrm{b},-\mathrm{c} \rightarrow+\mathrm{c},-\mathrm{e} \rightarrow+\mathrm{e},-\mathrm{f} \rightarrow+\mathrm{f}$, and $-\mathrm{g} \rightarrow+\mathrm{g}$.

For $\mathrm{CP}_{2}$, we can obtain event sequences satisfying the above constraints in a standard way using reachability tree. In Figure 4 , notation $(x, y, z)$ means a global state where $x, y$ and $z$ of $P_{1}, P_{2}$ and $P_{3}$, respectively, are integers representing sequential order which is the same value of ' $\mathrm{m}$ ' in Definition 2. The initial global state is $(0,0,0)$ and the final one is $(4$, $4,4)$ in this example. In the left side of the tree, we have three event sequences ending with $(4,4,4)$. Thus, there is a rectangle containing ' 3 '. A leaf node with a circle denotes a transition to an intermediate node having the same global state as the leaf node. We have 36 event sequences in total as the result of summing up the values inside the rectangles.

Now let us construct a minimal length time-event sequence diagram to represent the above causal relationships.

Definition 4: A Minimal Causality Path (MCP) corresponding to a $\mathrm{CP}$ is a minimal length of Global Event sequences.

Minimal Causality Paths can be obtained in the same way that outlined in Section 3.2. The way is to calculate the linear logical time of events in a CP, and then obtain GEs for each logical time by collecting all events having the same logical time. Then MCP is a sequence of GEs aligned in the increasing order of logical time. For $\mathrm{CP}_{2}$, we obtain an MCP corresponding to Figure 2. If we attach an identifier of FSM to an event as a suffix in case of causing ambiguity, then

$\mathrm{MCP}_{2}=-\mathrm{a} \rightarrow(-\mathrm{b},+\mathrm{a}) \rightarrow\left(-\mathrm{c}_{1},+\mathrm{b}\right) \rightarrow+\mathrm{c}_{3} \rightarrow-\mathrm{e} \rightarrow+\mathrm{e} \rightarrow-\mathrm{f} \rightarrow(+\mathrm{f},-\mathrm{g}) \rightarrow+\mathrm{g}$.

In the same way, we can obtain MCPs corresponding to CPs as follows:

$\mathrm{MCP}_{1}=-\mathrm{a} \rightarrow(-\mathrm{b},+\mathrm{a}) \rightarrow+\mathrm{b} \rightarrow-\mathrm{c}_{3} \rightarrow+\mathrm{c}_{1} \rightarrow-\mathrm{d} \rightarrow+\mathrm{d} \rightarrow-\mathrm{f} \rightarrow(+\mathrm{f},-\mathrm{g}) \rightarrow+\mathrm{g}$.

$\mathrm{MCP}_{3}=-\mathrm{a} \rightarrow(-\mathrm{b},+\mathrm{a}) \rightarrow+\mathrm{b}$.

$\mathrm{MCP}_{4}=-\mathrm{a} \rightarrow(-\mathrm{b},+\mathrm{a}) \rightarrow\left(-\mathrm{c}_{1},+\mathrm{b}\right) \rightarrow-\mathrm{c}_{3}$.

$\mathrm{MCP}_{5}=-\mathrm{a} \rightarrow(-\mathrm{b},+\mathrm{a}) \rightarrow+\mathrm{b} \rightarrow-\mathrm{c}_{3} \rightarrow+\mathrm{c}_{1} \rightarrow-\mathrm{d}$.

$\mathrm{MCP}_{6}=-\mathrm{a} \rightarrow(-\mathrm{b},+\mathrm{a}) \rightarrow\left(-\mathrm{c}_{1},+\mathrm{b}\right) \rightarrow+\mathrm{c}_{3} \rightarrow-\mathrm{e}$.

The notation $(-b,+a)$ means that the two events occurred concurrently or mutually independently. Therefore, the order of occurrences of the two events does not matter. In addition, event ' $+\mathrm{a}$ ' could have occurred in the following circles spaced by synchronization delays in Figure 2 if a unit time assumption is relaxed to any natural numbers. In other words, event ' $+a$ ' is concurrent with events such as ' $-b$ ', ' $+b$ ', ' $-c$ ', ' $+c$ ', and '-e' which could be aligned with event ' $+a$ ' itself and the synchronization delays of event ' $+a$ ' in Figure 2.

\subsection{Generation of Combined Minimal Causality Path}

Combined Minimal Causality Path (CMCP) is a global event-sequence diagram such that each of its paths is associated with a corresponding MCP. A CMCP is generated so that each global transition is associated with a set of events, which can be implemented 


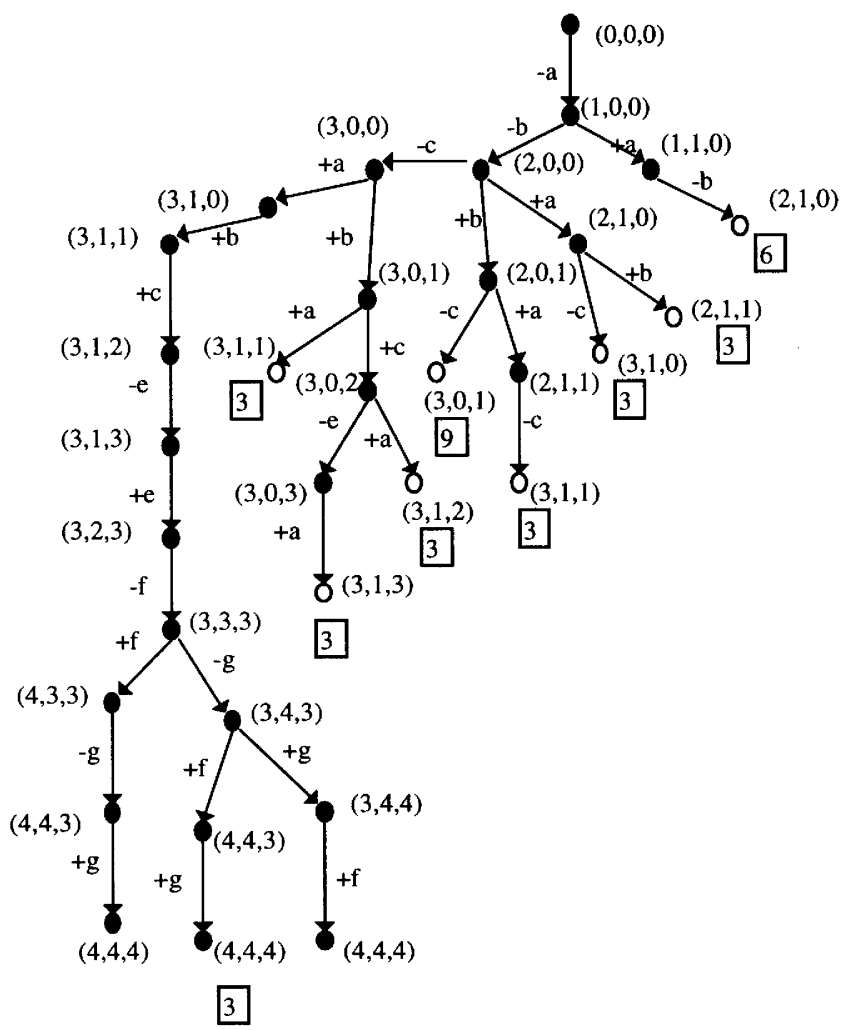

Figure 4 Generation of event sequences using reachability tree.

in parallel at the starting global state. Figure 5 shows the CMCP for the functional specification of Figure 3. The CMCP includes four MCP's described in Section 4.3. Four filled circles with the number $i$ showing $\mathrm{MCP}_{\mathrm{i}}$ in Figure 5 represent deadlocks.

The CMCP analysis is equivalent to the conventional reachability tree analysis while the computation times are enormously reduced. In order to measure the extent of reduction in our approach over the conventional way, let us show how many event sequences are represented by CPs. Using the same way that we obtained 36 event sequences for $\mathrm{CP}_{2}$, we have $18,3,15,6$ and 12 event sequences for $\mathrm{CP}_{1}, \mathrm{CP}_{3}, \mathrm{CP}_{4}, \mathrm{CP}_{5}$, and $\mathrm{CP}_{6}$, respectively. On the whole, the six CPs represent 90 event sequences. A CP is equivalent to an MCP under the assumption of unit logical time. If the number and the complexity of subsystems increase, the saving of testing effort becomes tremendous. For instance, 6 CPs represent over 10,000 event sequences in the example in (Kajiw, 1985) dealing with a concurrent system (modeled as CFSMs) consisting of two processors and two terminals of which numbers of states are $26,14,17$ and 7 respectively. 


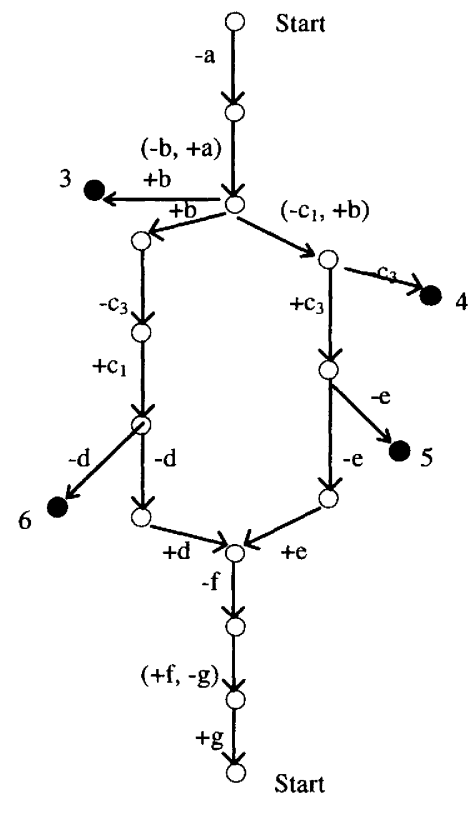

Figure 5 CMCP of the concurrent system in Figure 3.

\subsection{Test cases generation and application}

Test cases for a concurrent system are generated based on a specific test architecture. We view a concurrent system as a closed system which includes subsystems, the environment and operators if any. We assume that the external behavior of each subsystem in the concurrent system can be observed through one or more Point of Observations (POs) between subsystems. Subsystems under test are regarded as blackboxes. Subsystems not under test could include subsystems exposed as whiteboxes such as testers and operators.

In Figure 6 (a), we observe a trace and then decide whether the trace conforms to the system specifications. The CMCP given in Figure 5 is the minimal causality relation to which the trace should conform. Note the following characteristics of PO. Observing a message through PO implies that a message send event has occurred, but the corresponding receive event has not occurred yet. Therefore, in order to decide whether the receive event has actually happened, we need to wait for the subsequent observable events at the subsystem where the receive event is to occur. In reality, it is very hard to guess when the receive event has happened. For the time being, let us define Observability Rate as follows: Definition 5: Observability Rate (OR) is the number of observable events divided by the total number of events in a computation.

Note that the computation in Definition 5 can be either the partial or total behaviors of a system. Because we can observe all message send events at the POs in the case of the 


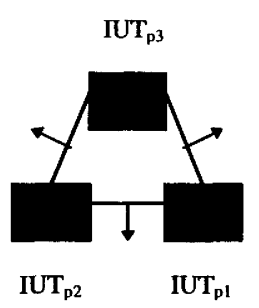

(a)

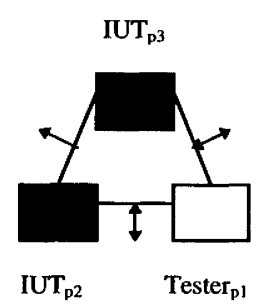

(b)

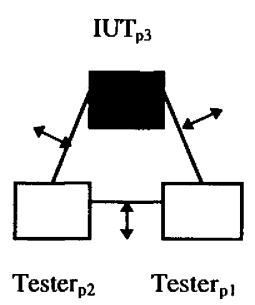

(c)

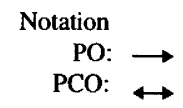

Figure 6 Test architecture.

computation shown in Figure 2 using the test architecture of Figure 6 (a), OR for Figure 6 (a), $\mathrm{OR}_{\mathrm{a}}$ for $\mathrm{CP}_{2}$, is $6 / 12$.

Definition 6: A Stable State (SS) is a global state of a system in which the system can not proceed without inputs from the subsystems not under test.

Some of the subsystems may be testers as in (b) and (c). In the case of (b), we need to project the test cases obtained in the previous section with respect to $P_{1}$. Also, for the architecture of (c), projecting the test cases with respect to $P_{2}$ is needed as well. Because Tester $_{\mathrm{pl}}$ in case (b) controls inputs such as ' $-\mathrm{a}$ ', ' $-\mathrm{b}$ ', ' $-c_{1}$ ' and '-d' in addition to POs in (a), we can test $P_{2}$ and $P_{3}$ to see whether they conform to their specifications. Therefore, we have SSs in the event sequence: $-a \rightarrow(-b,+a) \rightarrow-d$ and $-a \rightarrow(-b,+a) \rightarrow\left(-c_{1},+b\right)$. If you look at $P_{1}$ in Figure 3, you can understand the SSs easily. Let us work at the SS in the event sequence $-a \rightarrow(-b,+a) \rightarrow\left(-c_{1},+b\right)$. Tester ${ }_{p 1}$ controls inputs such as ' $-a$ ', '-b' and ' $-c$ ', and

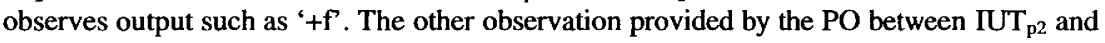
IUT $_{\mathrm{p} 3}$ gives us some information described in the architecture (a). In case of the computation shown in Figure 2, $\mathrm{OR}_{b}$ for $\mathrm{CP}_{2}$ is $7 / 12$ because of ' $+\mathrm{f}$ ' which is not counted in $\mathrm{OR}_{\mathrm{a}}$ for $\mathrm{CP}_{2}$.

In case (c), Tester $_{\mathrm{p} 1}$ and Tester $_{\mathrm{p} 2}$, with more PCOs, provide us more SSs. The SS of IUT $_{p 2}$ is the event sequence: $-f \rightarrow-g$. OR $R_{c}$ of the computation in Figure 2 is $9 / 12$, because of ' $+a$ ' and ' $+\mathrm{e}$ '. Therefore, the architecture (c) provides more confidence in testing than the architecture (b). If we count all CPs, the difference of OR of the three architectures becomes significant. Also, we have the advantage of being able to test wrong behavior of IUTs which was not possible in (a).

Definition 7: Controllability Rate (CR) of a system under test is defined as $\sum_{i=1}{ }^{m} \mid$ Input $\left.\right|_{i}$ $/ \sum_{j=1}{ }^{n} \mid$ Input $\left.\right|_{j}$ where $m$ is the number of stable states of the system, $n$ is the number of stable states of a white-box system corresponding to the system, and $\mid$ Input $\left.\right|_{i}$ and $\mid$ Input $\left.\right|_{j}$ are the number of allowed inputs at the $\mathrm{i}$-th and $\mathrm{j}$-th Stable States respectively.

According to Definition 7, we obtain $\mathrm{CR}_{\mathrm{a}}=0 /(9 \times 1), \mathrm{CR}_{\mathrm{b}}=(4 \times 1) /(9 \times 1)$, and $\mathrm{CR}_{\mathrm{c}}$ $=(6 \times 1) /(9 \times 1)$. The higher $\mathrm{CR}$ is, the more controllable the system is. For example, in Figure 6, architecture (c) is more controllable than (b), and (b) is more controllable than (a). 
Let us discuss briefly how to relax the assumptions about this model in order to make it more applicable to the real world situation. Note the value of ' $d$ ' in logical clocks for representing the duration of an operation in Section 3.1. So far, we have assumed that the value of ' $d$ ' is one unit time. If the value ' $d$ ' can be relaxed to any natural number or any real number, then this model has wider applicability.

\section{CONCLUSION}

In order to test and verify concurrent behavior of protocols running in a concurrent system, traditional approaches are to map the multiple modules into a single module and analyze the sequential behavior of the resultant single module. Other approaches are to use interleaving methods for the events in a concurrent system. Recently some attempts have been made to adopt a causality method or a true concurrency model in protocol verifications and testing for concurrent systems (Probs, 1991, Ulric, 1995).

By adopting a causality relation model, we proposed a non-heuristic approach adopting true concurrency model for testing asynchronous communicating systems as treating them blackboxes. The approach is succinct and formal compared with the previous work (Araka, 1991, Kajiw, 1985), deals with the unintentional nondeterminism either arising from the concurrent environment or due to the blackbox approach. Also, the approach avoids the state space explosion problem by representing a number of event sequences as a class, which we call Minimal Causality Path using logical clocks. The relationship between test case generation and application in a concurrent system was also discussed. Also new definitions such as Observability Rate, Stable State, and Controllability Rate were introduced to quantify some aspects of the systems. Our model is adequate to be a base model to investigate further work in this area.

For further study, we are working on enhancement of the model as follows:

- relaxing of the logical unit time to any natural number,

- resolving of the intentional nondeterminism in the specification of concurrent systems, and

- minimization of "state space explosion" in generating Concurrent Paths.

\section{ACKNOWLEDGEMENT}

The authors would like to thank anonymous reviewers for their helpful comments in revising this paper.

\section{REFERENCES}

Arakawa, N. and Soncoka, T. (1991) "A Test Case Generation Method for Concurrent Programs," Proceedings of the Int'l Workshop on Protocol Test Systems.

Cavalli, A., Kim, S. U., and Maigron, P. (1993) "Improving Conformance Testing for LOTOS," pp. 371 - 386, Sixth Int'l Conference on Formal Description Techniques. 
Charron-Bost, B. (1989) "Combinatories and Geometry of Consistent Cuts: Application to Concurrency Theory," The 3rd Int'l Workshop on Distributed Algorithms, LNCS 392, Springer-Verlag, pp. 45 - 56.

Fidge, C. (1991) "Logical Time in Distributed Computing Systems," IEEE Computer, pp. $28-33$.

ISO IS 9646 (1994) OSI Conformance Testing Methodology and Framework.

Kajiwara, M., Ichikawa, H., Itoh, M., and Yoshida, Y. (1985) "Specification and Verification of Switching Software," IEEE Tr. on Communications, pp. 193 - 198.

Kim, M. C., Chanson, S. T., and Vuong, S. T. (1993) "Concurrency Model and its Application to Formal Protocol Specifications," IEEE INFOCOM, pp. 766 - 773.

Kim, M. C., Chanson, S. T., Yoo, S. J. (1995) "Design for Testability for Protocols based on Formal Specifications," Proceedings of the 8th Int'l Workshop on Protocol Test Systems.

Lamport, L. (1978) "Time, Clocks, and the Ordering of Events in a Distributed System," CACM, pp. 558 - 565.

Lee, D., Sabnani, K. K., Kristol, D. M. and Paul, S. (1993) "Conformance Testing of Protocols Specified as Communicating FSMs," IEEE INFOCOM, pp. 115 - 127.

Luo, G., Bochmann, G. V., and Petrenko, A. (1994) "Test Selection Based on Communicating Nondeterministic Finite-State Machines Using a Generalized WpMethod," IEEE Tr. on Software Engineering, pp. 149 - 161.

Mattern, F. (1988) "Virtual Time and Global States of Distributed Systems," Parallel and Distributed Algorithms, pp. 215 - 226, North-Holland.

Probst, D. K. and Li, H. F. (1991) "Using Partial-order Semantics to Avoid the State Explosion Problem in Asynchronous Systems," 2nd Int'l Conference on Computeraided Verification.

Raynal, M. (1992) "About Logical Clocks for Distributed Systems," ACM Operating Systems Review, pp. 41 - 48.

Raynal, M., Mizuno, M., and Neilsen, M. L. (1992) "Synchronization and Concurrency Measures for Distributed Communications," $12^{\text {th }}$ Int'l Conference on Distributed Computing Systems, pp. 700 - 707.

Taylor, R. N., Levine, D. L., and et al. (1992) "Structural Testing of Concurrent Programs," IEEE Tr. on Software Engineering, pp. 206 - 215.

Tai, K. C. and Carver, R. H. (1995) "Testing of Distributed Programs," Handbook of Parallel and Distributed Computing, ed. A. Zomaya, McGraw Hill.

Ulrich, A. and Chanson, S. T. (1995) "An Approach to Testing Distributed Software Systems," Int'l Symposium on Protocol Specifications, Testing, and Verification.

Verhaad, L., Tremans, J., Kars, P. and Brinksma, E. (1992) "On Asynchronous Testing," Int'l Workshop on Protocol Test Systems, pp. 14 - 27.

Vissers, C. (1990) "FDTs for Open Distributed Systems, A Retrospective and A Prospective View," Int'l Symposium in Protocol Specifications, Testing and Verification.

Young, M., and Taylor, R. N. (1988) "Combining Static Concurrency Analysis with Symbolic Execution," IEEE Tr. on Software Engineering, pp. 1499 - 1511. 


\section{BIOGRAPHY}

Myungchul Kim received B.A. in electronics engineering from Ajou Univ. in 1982, M.S. in computer science from the Korea Advanced Institute of Science and Technology in 1984, and $\mathrm{Ph} . \mathrm{D}$. in computer science from the Univ. of British Columbia in 1992. Currently, he is with the Korea Telecom Research and Development Group as a managing director, chairman of Profile Test Specification - Special Interest Group of Asia-Oceania Workshop, and will be chair of the 10th Int'l Workshop on Testing of Communicating Systems. His interests include protocol engineering on telecommunications and multimedia.

Samuel T. Chanson received his Ph.D. degree in Electrical Engineering and Computer Sciences from the University of California, Berkeley in 1975 . He was a faculty member at the School of Electrical Engineering, Purdue University for two years before joining the Department of Computer Science at the University of British Columbia where he was a professor and director of its Distributed Systems Research Group. In 1993 he joined the Hong Kong University of Science \& Technology as professor and Associate Head of the Computer Science Department. He has consulted widely for industry and government on communication technologies. He has published about 100 technical papers in journals and conference proceedings, and has served on the program committee of many international conferences on distributed systems and computer communications. He was the conference cochair of the first IFIP International Workshop on Protocol Test Systems (now called IWTCS), a co-chair of the 1994 International Conference on Protocol Specification, Testing and Verification, and the program co-chair of the IEEE International Conference on Distributed Computing Systems in May 1996. Dr. Chanson's research interests include protocol testing, ATM network management, multimedia applications, and Internet technologies. $\mathrm{He}$ is the director of Cyberspace Centre for Hong Kong software industry.

Sungwon Kang received a B.A. degree from Seoul National University in Korea in 1982 and received his M.S. and Ph.D. in computer science from the University of Iowa in U.S.A in 1989 and 1992. Since 1993, he has been a senior researcher at Korea Telecom Research and Development Group. Currently he is a guest researcher at National Institute of Standards and Technology of U.S.A. His research interests include communication protocol testing, program optimization and programming languages.

Jaehwi Shin received the B.S. and M.E. degrees in electronic engineering from Hanyang University and Seoul National University in 1991 and 1993, respectively. Currently he is with the Korea Telecom Research and Development Group as a member of Protocol Engineering Team. His research interests include intelligent networks, communication protocol testing, digital signal processing and circuit theory. 